\title{
Obstructed defecation: the role of anorectal manometry
}

\author{
F. Pucciani - M. N. Ringressi
}

Received: 13 July 2011/Accepted: 29 November 2011/Published online: 16 December 2011

(C) Springer-Verlag 2011

\begin{abstract}
Background The aim of the study was to evaluate the clinical usefulness of anorectal manometry (AM) in patients affected by obstructed defecation (OD).

Methods Between January 2007 and December 2010, 379 patients (287 women and 92 men) affected by OD were evaluated. After a preliminary clinical evaluation, defecography and AM were performed. The results were compared with those from 20 healthy control subjects.

Results Overall anal resting pressure was not significantly different between patients and controls. Maximal voluntary contraction (MVC) data were significantly lower when compared with those of controls $(P<0.01)$. The straining test was considered positive in 143 patients. No significant difference was noted between patients and controls in maximal tolerated volume data. Patients had a significantly higher conscious rectal sensitivity threshold than controls $(P<0.02)$.

Conclusions A positive straining test, low MVC and impaired rectal sensation are the main abnormalities detected by AM in patients with OD.
\end{abstract}

Keywords Obstructed defecation - Anorectal manometry · Chronic constipation $\cdot$ Rectal sensation

\section{Introduction}

Obstructed defecation (OD), as identified by Bartolo [1], is broadly defined as the inability to evacuate contents from the rectum [2] and is accompanied by symptoms of dyschezia

F. Pucciani $(\bowtie) \cdot$ M. N. Ringressi

Department of Medical and Surgical Critical Care,

University of Florence, Largo Brambilla 3,

50134 Firenze, Italy

e-mail: pucciani@unifi.it and a subjective sensation of anal blockage during defecation [3]. Outlet obstruction may be caused by organic or functional diseases and only a thorough diagnostic workup can identify the causes. Mechanical causes include rectocele, rectoanal intussusception, descending perineum syndrome, solitary rectal ulcer syndrome, mucosal rectal prolapse, enterocele and sigmoidocele. The functional diseases include disorders of rectal sensation and pelvic floor dyssynergia [4]. The diagnostic workup of patients with obstructed defecation includes morphologic techniques, such as defecography and magnetic resonance imaging (MRI), to evaluate anatomical abnormalities of the anorectum and pelvis during evacuation, functional techniques, such as anorectal manometry (AM) and neurophysiological tests (sphincter electromyography, latency of the bulbocavernosus reflex, evoked potentials), to evaluate motor and sensory dysfunction of the anorectum. At the present time, $\mathrm{AM}$ is extensively used to identify the pathophysiological mechanisms of anorectal obstruction in constipated patients but its clinical usefulness is debated. Rasmussen et al. reported that standard AM did not show any differences between constipated patients and controls [5], whereas many others state that AM is a necessary diagnostic step in chronic constipation [6-8]. Otherwise the role of AM in the evaluation of patients with OD is not well defined.

The purpose of this study was to evaluate the clinical usefulness of AM in patients affected by obstructed defecation in order to identify manometric profiles that may be helpful in choosing the correct treatment.

\section{Materials and methods}

Between January 2007 and December 2010, 433 patients affected by OD with negative colonoscopy were seen at the 
outpatient unit of the Surgery Clinic of the University of Florence. All patients were entered into a prospectively constructed database, which contained 1,703 patients at the time of the study. Exclusion criteria for the study were as follows: age older than 80 years, impaired general health, neurological disease, physical handicap, general problems (language, distance from the outpatient unit, non-collaboration). Fifty-four patients were excluded from analysis: 16 patients were over 80 years, 8 were affected by advanced pulmonary diseases, 14 had a neurological disease, 5 a physical handicap and 11 had other general problems.

The remaining 379 patients [287 women with median age of 51.2 years (range 19-73) and 92 men with median age of 54.0 years (range 23-70)] were included in the study. All patients received a preliminary clinical evaluation and were studied by means of defecography and anorectal manometry.

Their results were compared with clinical and anorectal manometric data obtained from healthy control subjects. The control group consisted of 20 healthy volunteers [12 women with median age of 53.4 years (range 44-69); 8 men with median age of 52.8 years (range 37-63)] with normal bowel habits. They perceived their defecation behavior as normal and had never visited a physician for intestinal problems.

In accordance with the ethical guidelines of our university, all of the participants provided written consent to participate in the study with full knowledge of the procedures to be undertaken.

\section{Clinical evaluation}

All patients received a clinical evaluation. Information regarding bowel movements according to the Rome Criteria III [9] and pathologic conditions were noted. We recorded previous pelvic and/or anal surgery, and, in women, deliveries, noting obstetric tears and episiotomy, and degree of genital relaxation assessed with the Pelvic Organ Prolapse Quantification system (POP-Q) [10]. From December 2008, in all patients, obstructed defecation was classified according to the obstructed defecation syndrome (ODS) score [11].

\section{Defecography}

All patients underwent defecography, according to the methods suggested by the Italian working team [12]. Defecography was performed with the patient at rest, during squeeze and during expulsion of the barium. All the radiological measurements were taken in the later view. The radiological measurements included the anorectal angle (ARA) and pelvic floor descent. Qualitative evaluation was made by noting rectocele, rectoanal intussusception and persistence of the puborectalis indentation during evacuation. Attention was paid to the presence of enterocele, sigmoidocele or megarectum.

\section{Anorectal manometry}

All patients underwent AM using our standard protocol [13]. The recordings and the analyses of the tracings were made by a computerized system (Dyno Compact, Menfis bioMedica $^{\circledR}$ s.r.l., Bologna, Italy). Anal resting pressure (ARP) was measured four times and recorded in mmHg. The stationary pull-through technique was used. Computerized analysis identified the maximal anal pressure $\left(P_{\max }\right.$; normal range $\geq 50$ and $\leq 150 \mathrm{mmHg}$ ) and the mean pressure $\left(P_{\mathrm{m}}\right.$; normal range $\geq 20$ and $\left.\leq 60 \mathrm{mmHg}\right)$ of the anal canal. The maximal voluntary contraction (MVC) was evaluated by asking the subject to voluntarily contract the anal sphincter as long as he/she could. The computer quantified the amplitude in $\mathrm{mmHg}$ and duration in seconds. The rectoanal inhibitory reflex (RAIR) was elicited by inflating a soft rubber balloon in the rectum at $10 \mathrm{~cm}$ from the anal verge: the volume was increased every $20 \mathrm{ml}$ according to Martelli et al. [14] The first distension volume at which internal sphincter relaxation occurred (RAIR threshold) and the distension volume for which an initial transient sensation occurred [conscious rectal sensitivity threshold (CRST)] were determined in all patients. The maximal tolerated volume (MTV) was also measured in all subjects: it was considered an expression of rectal reservoir capacity. Compliance of the rectum (expression of the ratio $\mathrm{mmHg} / \mathrm{ml}$ of inflated air) was detected by means of the pressure/volume curve. A low pressure/volume curve was defined when $\triangle \mathrm{P} / \mathrm{DV}$ was $<0.1$ for each point of curve. The manometric procedure ended by measuring anal pressures during attempted defecation (straining test). The straining test was considered positive if an inappropriate increase or less than $20 \%$ relaxation of basal resting pressure occurred.

\section{Statistical analysis}

The results are expressed as the mean \pm standard deviation (SD). Student's $t$ test for paired and unpaired samples was used for statistical analyses. All correlations were evaluated using the Spearman's rank correlation coefficient (rho: $\rho_{\mathrm{s}}$ ), where full correlation is 1 and correlation $<0.50$ is considered not significant. A $P<0.05$ was chosen for rejection of null hypothesis.

\section{Results}

Table 1 shows the clinical characteristics of all 379 patients. No statistical differences were found regarding the number 
Table 1 Clinical evaluation

\begin{tabular}{lll}
\hline & Patients (379) & Control subjects (20) \\
\hline Vaginal deliveries $^{\mathrm{a}}$ & $1.38 \pm 0.8$ & $1.45 \pm 0.3$ \\
Obstetric tears $^{\mathrm{a}}$ & $107 / 287$ & $4 / 12$ \\
Episiotomy $^{\mathrm{a}}$ & $54 / 287$ & $2 / 12$ \\
Previous pelvic surgery & $49 / 379$ & $2 / 20$ \\
Previous anal surgery & $68 / 379^{*}$ & $0 / 20$
\end{tabular}

* Patients versus controls: $P<0.005$

a 287 female patients; 12 female controls

of deliveries, obstetric tears and episiotomies between the two groups. One hundred and seventeen $(30.8 \%)$ patients had undergone previous surgery. Thirty-one women $(10.8 \%)$ had undergone hysterectomy via the abdominal or vaginal approach. Five males had undergone trans urethral resection of the prostate. Thirteen patients had undergone miscellaneous pelvic surgery for rectal prolapse, pelvic organ prolapse, diverticulitis. Anal surgery had been performed in 68 patients $(17.9 \%)$, mainly for hemorrhoids $(12.6 \%)$. The degree of genital relaxation, obtained by means of the POP-Q examination, is reported in Table 2. Ninety-four female patients (32.7\%) showed some sign of genital prolapse. They had a higher incidence of any POP-Q stage compared with the control group $(P<0.01)$. Fifteen patients $(9$ in Stage 2 and 6 in Stage 3) had a high ODS score ( $>24)$. No significant difference in daily stool frequency was found between all patients $(0.78 \pm 0.2)$ and controls $(1.2 \pm 0.4)$. The mean ODS score in 206 patients was $15.7 \pm 4.6$ while 21 patients $(10.1 \%)$ had an ODS score $\geq 24$. Correlations between clinical findings and the ODS score are reported in Table 3. There was no significant correlation between ODS score and stool frequency $\left(\rho_{\mathrm{s}} 0.16\right), \mathrm{POP}-\mathrm{Q}\left(\rho_{\mathrm{s}} 0.35\right)$ and previous anal surgery $\left(\rho_{\mathrm{s}}\right.$ 0.19). A significant correlation was found between ODS score and previous pelvic surgery $\left(\rho_{\mathrm{s}} 0.69\right.$; $P<0.05)$. Defecographic data are reported in Table 4 . The pelvic floor descent values in patients were significantly

Table 2 Pelvic organ prolapse quantification (POP-Q) in patients and control subjects

\begin{tabular}{lll}
\hline Stage & Patients $(287)$ & Control subjects (12) \\
\hline 0 & $193 / 287$ & $10 / 12$ \\
1 & $69 / 287$ & $2 / 12$ \\
2 & $17 / 287$ & $0 / 12$ \\
3 & $8 / 287$ & $0 / 12$ \\
4 & $0 / 287$ & $0 / 12$ \\
\hline
\end{tabular}

Stage 0 , no prolapse; Stage 1, leading edge of the prolapse is $>1 \mathrm{~cm}$ above the hymen; Stage 2, leading edge of the prolapse is $\leq 1 \mathrm{~cm}$ proximal or distal to the plane of the hymen; Stage 3, leading edge of the prolapse is $>1 \mathrm{~cm}$ below the plane of the hymen but protrudes no more than $2 \mathrm{~cm}$ less the total vaginal length; Stage 4, essentially complete eversion of the total lower genital tract
Table 3 Correlations (Spearman's coefficient: $\rho_{\mathrm{s}}$ ) among clinical reports and obstructed defecation syndrome (ODS) scores in 379 patients

\begin{tabular}{ll}
\hline Clinical reports & $\rho_{\mathrm{s}}$ \\
\hline Stool frequency & 0.16 \\
Pelvic organ prolapse & 0.35 \\
Previous pelvic surgery & $0.69^{*}$ \\
Previous anal surgery & 0.19 \\
\hline
\end{tabular}

$* P<0.05$

Table 4 Defecographic data

\begin{tabular}{lll}
\hline & Patients & Control subjects \\
\hline Anorectal angle $\left(^{\circ}\right)$ & & \\
$\quad$ Resting & $95.7 \pm 9.2$ & $94.5 \pm 3.1$ \\
$\quad$ Evacuation & $104.1 \pm 11.2$ & $110.1 \pm 3.4$ \\
Pelvic floor descent (mm) & & \\
Resting & $26.1 \pm 11.7^{*}$ & $17.3 \pm 7.2$ \\
Evacuation & $41.7 \pm 17.3^{*}$ & $25.2 \pm 2.5$ \\
Rectocele & & \\
Affected patients & $223 / 379$ & 0 \\
Size (mm) & $24.7 \pm 9.2$ & 0 \\
Rectoanal intussusception & & \\
$\quad$ Affected patients & $127 / 379$ & 0 \\
Puborectal indentation & & 0 \\
$\quad$ Affected patients & $136 / 379$ & 0 \\
Enterocele & $18 / 379$ & 0 \\
Affected patients & & \\
Sigmoidocele & & \\
Affected patients & $6 / 379$ & \\
Megarectum & $7 / 379$ & \\
Affected patients & 0.01 & \\
\hline
\end{tabular}

* Patients versus controls: $P<0.01$

higher at rest and during evacuation $(P<0.01)$ than in the controls. One hundred and fifty patients had a decreased ARA opening at evacuation and 136 patients $(35.8 \%)$ had puborectalis indentation. They were considered to be affected by pelvic floor dyssynergia according to the coexistence of manometric positive straining test. Rectoanal intussusception was noted in 127 (33.5\%) of all patients and in 77 of these $(60.6 \%)$, the rectoanal intussusception was combined with rectocele. Overall, rectocele was present in 223 patients $(58.8 \%)$. Eighteen patients showed signs of enterocele, 6 had sigmoidocele, and megarectum was found in 7 patients. Table 5 shows ODS scores according to radiological diagnostic findings. Table 6 shows the distribution of anal resting pressure $\left(P_{\max }\right.$ and $\left.P_{\mathrm{m}}\right)$ and MVC (MVC-P: amplitude; MVC-T: duration). Overall anal resting pressure was not significantly different between patients and controls. However, AM identified a low $P_{\max }\left(P_{\max }<50 \mathrm{mmHg}\right)$ in 
Table 5 Radiological findings and obstructed defecation syndrome (ODS) scores

\begin{tabular}{ll}
\hline & ODS scores \\
\hline Total patients $(N=379)$ & $15.7 \pm 4.6$ \\
Pelvic floor dyssynergia $(N=136)$ & $14.9 \pm 4.8$ \\
Rectocele $(N=223)$ & $15.5 \pm 5.2$ \\
Rectoanal intussusception $(N=127)$ & $16.6 \pm 4.5$ \\
\hline
\end{tabular}

Table 6 Anal pressure data

\begin{tabular}{llc}
\hline & All patients (379) & Control subjects (20) \\
\hline$P_{\max }(\mathrm{mmHg})$ & $80.3 \pm 25.2$ & $88.2 \pm 10.1$ \\
$P_{\mathrm{m}}(\mathrm{mmHg})$ & $38.7 \pm 13.3$ & $47.8 \pm 7.6$ \\
MVC-P $(\mathrm{mmHg})$ & $71.7 \pm 39.1^{*}$ & $200.0 \pm 20.0$ \\
MVC-T $(\mathrm{sec})$ & $18.8 \pm 11.9^{*}$ & $35.6 \pm 4.2$ \\
\hline
\end{tabular}

* Patients versus control subjects: $P<0.01$

$P_{\max }$ maximum resting pressure, $P_{m}$ mean resting pressure, $M V C-P$ maximum voluntary contraction amplitude, $M V C$ - $T$ maximum voluntary contraction duration

45 patients $(11.8 \%)$ and in none of controls $(P<0.03)$ while a high $P_{\max }\left(P_{\max }>110 \mathrm{mmHg}\right)$ was identified in 66 patients $(17.4 \%)$ and in 1 control $(5 \%)(P<0.001)$. Low $P_{\mathrm{m}}$ $\left(P_{\mathrm{m}}<20 \mathrm{mmHg}\right)$ was identified in 44 patients $(11.6 \%)$ and in 1 control $(5 \%)(P<0.001)$ while a high $P_{\mathrm{m}}\left(P_{\mathrm{m}}>\right.$ $60 \mathrm{mmHg}$ ) was identified in 22 patients $(5.8 \%)$ and in none of controls $(P<0.05)$.

In this way, on the whole, 96 patients $(25.3 \%)$ had an anal resting pressure profile that was significantly different from that of control subjects $(P<0.01)$. The correlation between POP-Q and mean anal resting pressure is reported in Table 7. A positive direct correlation was noted between stage 3 of POP-Q and low mean anal resting pressure $\left(\rho_{\mathrm{s}} 0.63 ; P<0.01\right)$. MVC amplitude and duration data were significantly lower when compared with those of controls $(P<0.01)$. The straining test was considered positive in 143 patients: an inappropriate increase in anal pressure was detected in 115 patients (80.4\%) while the other $28 \mathrm{had}$ less than $20 \%$ relaxation of basal resting pressure. The RAIR was detected in all patients. RAIR threshold, MTV, and rectal sensation data are reported in Table 8. No significant difference was noted between patients and controls for RAIR threshold and MTV data. Patients had a significantly higher CRST than controls $(P<0.02)$. The highest CRST values were detected in patients affected by megarectum $(211.8 \pm 42.9 \mathrm{ml})$. The same 7 patients had the highest RAIR threshold $(98.5 \pm 11.3 \mathrm{ml})$ and MTV (418.6 \pm $75.3 \mathrm{ml}$ ) values. Overall compliance of the rectum was not significantly different between patients and controls, even if
Table 7 Correlations (Spearman's coefficient: $\rho_{\mathrm{s}}$ ) between pelvic organ prolapse quantification (POP-Q) and mean anal resting pressure $\left(\mathrm{P}_{\mathrm{m}}\right)$ in 94 patients

\begin{tabular}{ll}
\hline POP-Q stage & $\rho_{\mathrm{s}}$ \\
\hline $1(69 \mathrm{pts})$ & 0.40 \\
$2(17 \mathrm{pts})$ & 0.48 \\
$3(8 \mathrm{pts})$ & $0.63^{*}$ \\
\hline$P<0.01$ &
\end{tabular}

Table 8 Rectoanal inhibitory reflex threshold (RAIRT), maximal tolerated volume (MTV) and conscious rectal sensitivity threshold (CRST)

\begin{tabular}{lcc}
\hline & Patients & Control subjects \\
\hline RAIRT (ml) & $42.8 \pm 4.7$ & $39.7 \pm 9.6$ \\
MTV (ml) & $187.3 \pm 27.4$ & $205.1 \pm 23.4$ \\
CRST (ml) & $76.8 \pm 31.3^{*}$ & $50.7 \pm 10.3$ \\
\hline
\end{tabular}

* Patients versus control subjects: $P<0.02$

76 patients ( 69 affected by rectocele and 7 by megarectum) had a low pressure/volume curve.

\section{Discussion}

Obstructed defecation is an "iceberg syndrome", because it is indicative of multiple anatomical and functional disorders which are both evident and occult, and each patient has more than one disorder $[15,16]$. A mix of organic and functional disorders may be detected, without having any specific relationship with symptoms and signs of OD. Because of this complexity, multiple diagnostic tools are required, one of which is AM that can provide data which can better define the pathophysiological mechanisms of OD. Although the wide range of normal values results in high variability of manometric data with a low negative predictive factor, our study presents a number of findings that support the usefulness of anorectal manometry. Measures of anal resting pressures can identify those patients (25.3\%) who exhibit a significant impairment of anal tone. For example, 44 patients $(11.6 \%)$ had a low mean anal resting pressure, and lower anal pressure profiles are positively related to grade 3 POP-Q $\left(\rho_{\mathrm{s}} 0.63 ; P<0.01\right)$. Nevertheless, anal hypertonia may be detected in some patients $(5.8 \%)$. These reports confirm the phenotypic variability of OD, suggested by Bharucha et al. [17]. One obvious consideration is that variations in anal resting pressure may influence therapeutic options because the treatment of a patient with OD who has an anal pressure profile that is borderline for fecal incontinence probably due to a sphincter lesion or to internal rectal mucosal 
prolapse [18] is different from that of one with anal hypertonia which suggests the presence of an underlying neurologic disease [19], anxiety disorder or phobic-anxiety disorder [20]. Impaired MVC was significantly present in our patients. The amplitude and duration of anal voluntary contraction were significantly lower $(P<0.01)$, meaning these patients had weak contractile activity of external anal sphincter and puborectalis muscle. This suggests that they had less strength in both muscles, perhaps because of a defective muscular unit recruitment or behavioral issues. In fact, the amplitude and duration of anal squeeze pressure are modified by rehabilitative treatment of obstructed defecation using biofeedback and/or pelviperineal kinesitherapy. This could be due to the positive influence of both rehabilitative techniques on the endurance-coordination of muscles [21, 22]. The detection of anal relaxation impairment during attempted defecation was an important manometric tool in 143 patients (37.7\%) since it identified dyssynergic defecation and allowed us to program appropriate rehabilitative treatment [3]. The paradoxical contraction or impaired ability to relax the pelvic floor muscles during defecation change the normal sequence of the pelvic floor muscle activity during any stage of the complex mechanism of defecation [4]. Typically, the manometric sign of the positive straining test disappears after a successful rehabilitation that removes this incoordination [3]. CRST data of patients were significantly different from those of control subjects $(P<0.02)$. A normal perception of a fecal bolus is determinant to triggering and maintaining defecation and CRST values higher than normal imply alterations in coordinated defecation. The subjective sensation is impaired in regard to starting the stimulus for defecation and also the perception of complete emptying of the rectum. Obviously the coordinated sensory-motor integration of the rectum is distorted and OD occurs. The importance of impaired CRST is evident in patients affected by megarectum in whom fecal impaction may occur as a consequence of a large rectum with a high volume of rectal sensation. The defective rectal sensory function, as suggested by high CRST, requires further evaluation to identify the level of impaired sensation. Receptors, afferent nerves or central processing might be involved and the use of other diagnostic studies is necessary (barostat, functional MRI, sensory-evoked potentials) to identify the cause of primary rectal hyposensitivity [23]. Indeed, the impaired rectal afferent pathway function could be due to pudendal neuropathy, possibly caused by vaginal deliveries or years of straining or both. Rectal compliance was modified in 79 of our patients (20.0\%), all of whom had low pressure/volume values $(<0.10 \mathrm{mmHg} / \mathrm{ml})$. This means the biomechanical properties of the rectal wall were defective and as a result tonic adaptation to endoluminal volumes was impaired. The distension of the rectal wall is passive, without resistance, impairing the volumetric sensation of the rectal content because the failure of a generation of forces and deformations in the rectal wall during distension does not stimulate rectal mechanoreceptors. In this way, a secondary rectal hyposensitivity takes place [23, 24]. All patients affected by megarectum had this manometric finding, with lowest pressure/volume values, showing an important dysfunction of the rectal wall.

\section{Conclusions}

There are several reasons to use AM in OD: (1) it can point to which pathophysiological mechanisms may be involved; (2) it provides measurements that may be integrated with those of other diagnostic techniques; (3) it generates useful data that assist in the selection of appropriate therapy.

Conflict of interest The authors have no conflict of interest to disclose.

\section{References}

1. Bartolo D, Roe A (1986) Obstructed defaecation. Br J Hosp Med 35:228-236

2. Khaikin M, Wexner SD (2006) Treatment strategies in obstructed defecation and fecal incontinence. World $\mathrm{J}$ Gastroenterol 28:3168-3173

3. Pucciani F, Raggioli M, Ringressi MN (2011) Obstructed defecation: what is the role of rehabilitation? Colorectal Dis. doi: 10.1111/j.1463-1318.2011.02644.x

4. Andromanakos N, Skandalakis P, Troupis T, Fillippou D (2006) Constipation of anorectal outlet obstruction: pathophysiology, evaluation and management. J Gastroenterol Hepatol 21:638-646

5. Rasmussen OO, Sørensen M, Tetzschener T, Christiansen J (1993) Dynamic anal manometry in the assessment of patients with obstructed defecation. Dis Colon Rectum 36:901-907

6. Azpiroz Z, Enck P, Whitehead WE (2002) Anorectal functioning testing: review of collective experience. Am J Gastroenterol 97:232-240

7. Siproudhis L, Eléouet M, Desformeaux V et al (2009) Diagnosis of dyschezia. Gastroenterol Clin Biol 33:F68-F74

8. Rao SSC, Singh S (2010) Clinical utility of colonic and anorectal manometry in chronic constipation. J Clin Gastroenterol 44:597-609

9. Bharucha AE, Wald A, Enck P, Rao S (2006) Functional anorectal disorders. Gastroenterology 130:1510-1518

10. Bump RC, Mattiasson A, Bø K et al (1996) The standardization of terminology of female pelvic floor dysfunction. Am J Obstet Gynecol 175:10-17

11. Altomare DF, Spazzafumo L, Rinaldi M et al (2008) Set-up and statistical validation of a new scoring system for obstructed defaecation syndrome. Colorectal Dis 10:84-88

12. Piloni V, Genovesi N, Grassi R et al (1993) National working team report on defecography. Radiol Med 85:784-793 [article in Italian]

13. Pucciani F, Rottoli ML, Bologna A et al (1996) Anterior rectocele and anorectal dysfunction. Int J Colorectal Dis 11:1-9

14. Martelli H, Devroede G, Arhan P et al (1978) Some parameters of large bowel motility in normal man. Gastroenterology 75:612618 
15. Pescatori M (2003) Rectoceles and the "iceberg syndrome". Urodinamica 13:15-22

16. Pescatori M, Spyrou M, Pulvirenti d'Urso A (2006) A prospective evaluation of occult disorders in obstructed defecation using the "iceberg diagram". Colorectal Dis 8:785-789

17. Bharucha AE, Fletcher JG, Seide B et al (2005) Phenotypic variation in functional disorders of defecation. Gastroenterology 128:1199-1210

18. Hompes R, Harmston C, Wijffels $\mathrm{N}$ et al. (2011) Excellent response rate of anismus to Botulinum toxin if rectal prolapse misdiagnosed as anismus ("pseudoanismus") is excluded. Colorectal Dis. doi:10.1111/j.14631318.2011.02561.x

19. Jost WH, Schrank B, Herold A, Leiss O (1999) Functional outlet obstruction: anismus, spastic pelvic floor syndrome, and dyscoordination of the voluntary sphincter muscles. Definition, diagnosis, and treatment from the neurologic point of view. Scand J Gastroenterol 34:449-453
20. Bellini M, Rappelli L, Alduini P et al (2003) Pelvic floor dyssynergia and psychiatric disorders. Does the snake bite its tail? Minerva Gastroenterol Dietol 49:135-139

21. Hong Ho Y, Tan M, Su Goh H (1996) Clinical and physiologic effects of biofeedback in outlet obstruction constipation. Dis Colon Rectum 39:520-524

22. Pucciani F, Rottoli ML, Bologna A et al (1998) Pelvic floor dyssynergia and bimodal rehabilitation: results of combined pelviperineal kinesitherapy and biofeedback training. Int $\mathbf{J}$ Colorectal Dis 13:124-130

23. Gladman MA, Lunniss PJ, Scott SM, Swash M (2006) Rectal hyposensitivity. Am J Gastroenterol 101:1140-1151

24. Scott SM, van den Berg MM, Benninga MA (2011) Rectal sensorimotor dysfunction in constipation. Best Pract Res Clin Gastroenterol 25:103-118 